\title{
A connected 3-state reversible Mealy automaton cannot generate an infinite Burnside group *
}

\author{
Ines Klimann ${ }^{1}$, Matthieu Picantin ${ }^{1}$, and Dmytro Savchuk ${ }^{2}$ \\ 1 Univ Paris Diderot, Sorbonne Paris Cité, LIAFA, UMR 7089 CNRS, \\ F-75013 Paris, France \{klimann,picantin\}@liafa.univ-paris-diderot.fr \\ 2 Department of Mathematics and Statistics, University of South Florida, \\ 4202 E Fowler Ave - Tampa, FL 33620-5700, USA savchuk@usf.edu
}

\begin{abstract}
The class of automaton groups is a rich source of the simplest examples of infinite Burnside groups. However, there are some classes of automata that do not contain such examples. For instance, all infinite Burnside automaton groups in the literature are generated by non reversible Mealy automata and it was recently shown that 2-state invertible-reversible Mealy automata cannot generate infinite Burnside groups. Here we extend this result to connected 3-state invertiblereversible Mealy automata, using new original techniques. The results provide the first uniform method to construct elements of infinite order in each infinite group in this class.
\end{abstract}

Keywords and phrases Burnside groups, reversible Mealy automata, automaton groups

Digital Object Identifier 10.4230/LIPIcs.xxx.yyy.p

\section{Mealy automata and the General Burnside problem}

In 1902, Burnside has introduced a question which would become highly influential in group theory [6]:

Is a finitely generated group whose all elements have finite order necessarily finite?

This problem is now known as the General Burnside Problem. A group is commonly called a Burnside group if it is finitely generated and all its elements have finite order.

In 1964, Golod and Shafarevich [12, 13] were the first ones to give a negative answer to the general Burnside problem and around the same time Glushkov suggested that groups generated by automata could serve as a different source of counterexamples [11. In 1972, Aleshin gave an answer as a subgroup of an automaton group [2, and then in 1980, Grigorchuk exhibited the first and the simplest by now example of an infinite Burnside automaton group [14. Since then many infinite Burnside automaton groups have been constructed [4, 15, 16, 22. Even by now, the simplest examples of infinite Burnside groups are still automaton groups. All the examples of infinite Burnside automaton groups in the literature happen to be generated by non-reversible invertible Mealy automata, that is, invertible Mealy automata where all the letters do not act as permutations on the stateset.

It was proved in [17] that a 2-state invertible-reversible Mealy automaton cannot generate an infinite Burnside group, but the techniques were strongly based on the fact that the stateset has size 2. Here we address this problem for a larger class, namely the 3 -state invertible-reversible automata, and prove the following theorem.

* This work was partially supported by the french Agence Nationale pour la Recherche, through the Project MealyM ANR-JCJC-12-JS02-012-01. The third author was partially supported by the New Researcher Grant from USF Internal Awards Program.

() Ines Klimann, Matthieu Picantin, and Dmytro Savchuk;

licensed under Creative Commons License ND

Conference title on which this volume is based on.

Editors: Billy Editor, Bill Editors; pp. 1-12

Leibniz International Proceedings in Informatics

wr.... Schloss Dagstuhl - Leibniz-Zentrum für Informatik, Dagstuhl Publishing, Germany 
- Theorem 1. A connected 3-state invertible-reversible Mealy automaton cannot generate an infinite Burnside group.

For the proof of this theorem we develop new techniques, centered on the orbit tree of the dual of the Mealy automaton. We hope that these techniques could be further extended to attack similar problem for automata with bigger statesets.

The class of groups generated by automata is very interesting from algorithmic point of view. Even thought the word problem (given a word in generators decide if it represents the trivial element of the group) is decidable, most of other basic algorithmic questions, including finiteness problem, order problem, conjugacy problem, are either known to be undecidable, or their decidability is unknown. For example, it was proved recently that the order problem is undecidable for automaton semigroups [10] and for groups generated by, so called, asynchronous Mealy automata [5. However, this problem still remains open for the class of all groups generated by Mealy automata. There are many partial methods to find elements of infinite order in such groups, but the class of reversible automata is known as the class for which most of these algorithms do not work or perform poorly. The proof of Theorem 1 gives a uniform algorithm to produce many elements of infinite order in infinite groups generated by 3 -state invertible-reversible automata. Unfortunately, it does not provide an algorithm that can determine if the group itself is infinite, however, it is known that each invertible-reversible but not bireversible automaton generates an infinite group [1].

The paper is organized as follows. In Section 2, we set up notation, provide well-known definitions and facts concerning automaton groups and rooted trees. Certain results concerning connected components of reversible Mealy automata are given in Section 3 . In Section 4 we introduce a crucial construction for our proofs: the labeled orbit tree of a Mealy automaton. Finally, Section 5 contains the proof of our main results, including Theorem 1

\section{Basic notions}

\subsection{Groups generated by Mealy automata}

We first recall the formal definition of an automaton. A (finite, deterministic, and complete) automaton is a triple $\left(Q, \Sigma, \delta=\left(\delta_{i}: Q \rightarrow Q\right)_{i \in \Sigma}\right)$, where the stateset $Q$ and the alphabet $\Sigma$ are non-empty finite sets, and where the $\delta_{i}$ are functions.

A Mealy automaton is a quadruple $\left(Q, \Sigma, \delta=\left(\delta_{i}: Q \rightarrow Q\right)_{i \in \Sigma}, \rho=\left(\rho_{x}: \Sigma \rightarrow \Sigma\right)_{x \in Q}\right)$, such that both $(Q, \Sigma, \delta)$ and $(\Sigma, Q, \rho)$ are automata. In other terms, a Mealy automaton is a complete, deterministic, letter-to-letter transducer with the same input and output alphabet.

The graphical representation of a Mealy automaton is standard, see Figures 1 and 2 . A Mealy automaton $(Q, \Sigma, \delta, \rho)$ is invertible if the functions $\rho_{x}$ are permutations of $\Sigma$ and reversible if the functions $\delta_{i}$ are permutations of $Q$.

In a Mealy automaton $\mathcal{A}=(Q, \Sigma, \delta, \rho)$, the sets $Q$ and $\Sigma$ play dual roles. So we may consider the dual (Mealy) automaton defined by $\mathfrak{d}(\mathcal{A})=(\Sigma, Q, \rho, \delta)$. Obviously, a Mealy automaton is reversible if and only if its dual is invertible.

Let $\mathcal{A}=(Q, \Sigma, \delta, \rho)$ be a Mealy automaton. We view $\mathcal{A}$ as an automaton with an input and an output tape, thus defining mappings from input words over $\Sigma$ to output words over $\Sigma$. Formally, for $x \in Q$, the map $\rho_{x}: \Sigma^{*} \rightarrow \Sigma^{*}$, extending $\rho_{x}: \Sigma \rightarrow \Sigma$, is defined recursively by:

$$
\forall i \in \Sigma, \forall \mathbf{s} \in \Sigma^{*}, \quad \rho_{x}(i \mathbf{s})=\rho_{x}(i) \rho_{\delta_{i}(x)}(\mathbf{s}) .
$$




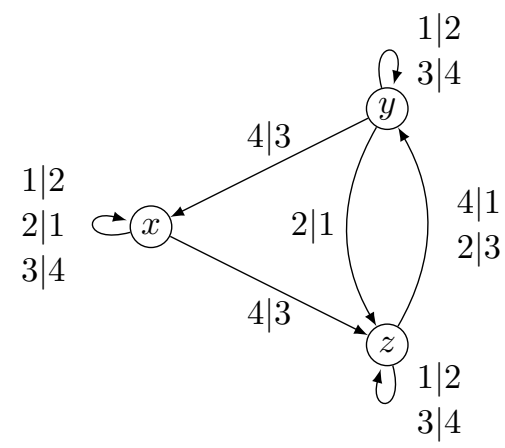

Figure 1 An example of a 4-letter 3-state connected Mealy automaton.

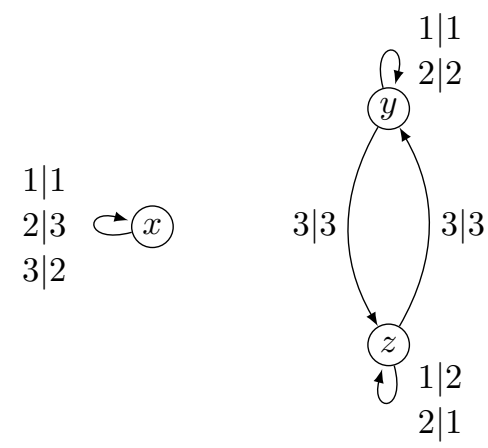

Figure 2 An example of a 3-letter 3-state disconnected Mealy automaton.

By convention, the image of the empty word is itself. The mapping $\rho_{x}$ for each $x \in Q$ is length-preserving and prefix-preserving. We say that $\rho_{x}$ is the production function associated with $(\mathcal{A}, x)$. For $\mathbf{x}=x_{1} \cdots x_{n} \in Q^{n}$ with $n>0$, set $\rho_{\mathbf{x}}: \Sigma^{*} \rightarrow \Sigma^{*}, \rho_{\mathbf{x}}=\rho_{x_{n}} \circ \cdots \circ \rho_{x_{1}}$.

Denote dually by $\delta_{i}: Q^{*} \rightarrow Q^{*}, i \in \Sigma$, the production functions associated with the dual automaton $\mathfrak{d}(\mathcal{A})$. For $\mathbf{s}=s_{1} \cdots s_{n} \in \Sigma^{n}$ with $n>0$, set $\delta_{\mathbf{s}}: Q^{*} \rightarrow Q^{*}, \delta_{\mathbf{s}}=\delta_{s_{n}} \circ \cdots \circ \delta_{s_{1}}$.

The semigroup of mappings from $\Sigma^{*}$ to $\Sigma^{*}$ generated by $\left\{\rho_{x}, x \in Q\right\}$ is called the semigroup generated by $\mathcal{A}$ and is denoted by $\langle\mathcal{A}\rangle_{+}$. When $\mathcal{A}$ is invertible, its production functions are permutations on words of the same length and thus we may consider the group of mappings from $\Sigma^{*}$ to $\Sigma^{*}$ generated by $\left\{\rho_{x}, x \in Q\right\}$. This group is called the group generated by $\mathcal{A}$ and is denoted by $\langle\mathcal{A}\rangle$.

Let us recall some known results that will be used in our proofs.

- Proposition 2 (see, for example, [1]). An invertible Mealy automaton generates a finite group if and only if it generates a finite semigroup.

- Proposition 3 ([1, 20, 21]). A Mealy automaton generates a finite (semi)group if and only if so does its dual.

As in the case of automata, there is a notion of minimization for Mealy automata: if several states have the same action, it consists of keeping only one of them. More formally, for a Mealy automaton $\mathcal{A}=(Q, \Sigma, \delta, \rho)$, the Nerode equivalence $\equiv$ on $Q$ is the limit of the sequence of increasingly finer equivalences $\left(\equiv_{k}\right)$ recursively defined by:

$$
\begin{aligned}
\forall x, y \in Q: \quad x \equiv_{0} y & \Longleftrightarrow \forall i \in \Sigma: \rho_{x}(i)=\rho_{y}(i) \\
\forall k \geqslant 0: x \equiv_{k+1} y & \Longleftrightarrow\left(x \equiv_{k} y \wedge \quad \forall i \in \Sigma: \delta_{i}(x) \equiv_{k} \delta_{i}(y)\right) .
\end{aligned}
$$

Since the set $Q$ is finite, this sequence is ultimately constant. The minimization of $\mathcal{A}$ is then the Mealy automaton $\mathfrak{m}(\mathcal{A})=(Q / \equiv, \Sigma, \tilde{\delta}, \tilde{\rho})$, where for every $(x, i)$ in $Q \times \Sigma$, we have $\tilde{\delta}_{i}([x])=\left[\delta_{i}(x)\right]$ and $\tilde{\rho}_{[x]}=\rho_{x}$.

If the minimizations of two invertible Mealy automata are structurally isomorphic, these automata generate two isomorphic groups. Moreover, the following lemma is straightforward:

- Lemma 4. Let $\mathcal{A}$ be an invertible Mealy automaton. If there exist $p<q$ such that $\mathfrak{m}\left(\mathcal{A}^{p}\right)$ and $\mathfrak{m}\left(\mathcal{A}^{q}\right)$ are isomorphic, then $\mathcal{A}$ generates a finite group. 


\subsection{Terminology on trees}

Throughout this paper, we will use different sorts of labeled trees. Here we set up some terminology that are common for all of them.

All our trees are rooted, i.e. with a selected vertex called the root. We will visualize the trees traditionally as growing down from the root. Hence, the "top" and "bottom" directions in the tree are defined as "to" and "from" the root respectively. It will be convenient to orient all edges in the tree in the direction from the root. The initial vertex of an edge $e$ is denoted by $T(e)$ and its terminal vertex by $\perp(e)$. A path is a (possibly infinite) sequence of adjacent edges without backtracking from top to bottom. A path is said to be initial if it starts at the root of the tree. A branch is an infinite initial path. The initial vertex of a non-empty path $\mathbf{e}$ is denoted by $T(\mathbf{e})$ and its terminal vertex by $\perp(\mathbf{e})$

The level of a vertex is its distance to the root and the level of an edge or a path is the level of its initial vertex. For $V$ a vertex in a tree $\mathcal{T}$, by section of $\mathcal{T}$ at $V$ (denoted by $\mathcal{T}_{\mid V}$ ) we mean the subtree of $\mathcal{T}$ with root $V$ consisting of all those vertices of $\mathcal{T}$ that are descendant of $V$. Additionally, for $\mathbf{e}$ an initial finite path of $\mathcal{T}$, we also call $\mathcal{T}_{\mid \perp(\mathbf{e})}$ the section of $\mathcal{T}$ at $\mathbf{e}$ and denote it by $\mathcal{T}_{\mid \mathbf{e}}$.

Whenever one considers a rooted tree whose edges are labeled by elements of a finite set, the label of a (possibly infinite) path is the ordered sequence of labels of the edges of this path.

\section{Powers of a Mealy automaton and their connected components}

In this section we detail the basic properties of the connected components of the powers of a reversible Mealy automaton. The link between these components is central in our construction.

Note that some of the notions below may be defined in a more general framework, but as in this paper we work only with reversible automata, we restrict our attention to this case in order to make the explanations easier.

Let $\mathcal{A}=(Q, \Sigma, \delta, \rho)$ be a reversible Mealy automaton. We will consider the connected components of the underlying graph of $\mathcal{A}$. By reversibility, all the connected components are strongly connected. One can also view the connected components of $\mathcal{A}$ as the orbits of the action of the group $\langle\mathfrak{d}(\mathcal{A})\rangle$ generated by the dual automaton on $Q$.

Now, in order to construct the orbit tree of $\mathfrak{d}(\mathcal{A})$, we will consider the connected components of the powers of $\mathcal{A}$ : for $n>0$, its $n$-th power $\mathcal{A}^{n}$ is the Mealy automaton

$$
\mathcal{A}^{n}=\left(Q^{n}, \Sigma,\left(\delta_{i}: Q^{n} \rightarrow Q^{n}\right)_{i \in \Sigma},\left(\rho_{\mathbf{x}}: \Sigma \rightarrow \Sigma\right)_{\mathbf{x} \in Q^{n}}\right) .
$$

By convention, $\mathcal{A}^{0}$ is the trivial automaton on a singleton stateset and the alphabet $\Sigma$.

The powers of the reversible Mealy automaton $\mathcal{A}$ are reversible and the connected components of $\mathcal{A}^{n}$ coincide with the orbits of the action of $\langle\mathfrak{d}(\mathcal{A})\rangle$ on $Q^{n}$.

- Definition 5. The connection degree of a Mealy automaton $\mathcal{A}$, denoted by $\curlywedge(\mathcal{A})$, is the largest $n$ such that $\mathcal{A}^{n}$ is connected. If $\mathcal{A}$ is not connected, its connection degree is 0 ; if all the powers of $\mathcal{A}$ are connected, its connection degree is infinite.

Note that a connection degree of $\mathcal{A}$ is infinite if and only if the group $\langle\mathfrak{d}(\mathcal{A})\rangle$ acts leveltransitively on the tree $Q^{*}$ (i.e. the action of $\langle\mathfrak{d}(\mathcal{A})\rangle$ on each level $Q^{n}$ of $Q^{*}$ is transitive). Since $\mathcal{A}$ is reversible, there is a very particular connection between the connected components of $\mathcal{A}^{n}$ and the connected components of $\mathcal{A}^{n+1}$ as highlighted in [17]. More precisely, suppose 
that $\mathcal{C}$ is a connected component of $\mathcal{A}^{n}$ for some $n$ and that $\mathbf{u} \in Q^{n}$ is a state of $\mathcal{C}$. Let also $x \in Q$ be a state of $\mathcal{A}$ and $\mathcal{D}$ be a connected component of $\mathcal{A}^{n+1}$ containing the state $\mathbf{u} x$. Then, for any state $\mathbf{v}$ of $\mathcal{C}$, there exists a state of $\mathcal{D}$ prefixed with $\mathbf{v}$ :

$$
\exists \mathbf{s} \in \Sigma^{*} \mid \delta_{\mathbf{s}}(\mathbf{u})=\mathbf{v} \quad \text { and so } \quad \delta_{\mathbf{s}}(\mathbf{u} x)=\mathbf{v} \delta_{\rho_{\mathbf{u}}(\mathbf{s})}(x) .
$$

Furthermore, if $\mathbf{u} y$ is a state of $\mathcal{D}$, for some state $y \in Q$ different from $x$, then $\delta_{\mathbf{s}}(\mathbf{u} x)$ and $\delta_{\mathbf{s}}(\mathbf{u} y)$ are two different states of $\mathcal{D}$ prefixed with $\mathbf{v}$, because of the reversibility of $\mathcal{A}^{n+1}$ : the transition function $\delta_{\rho_{\mathbf{u}}(\mathbf{s})}$ is a permutation.

Hence $\mathcal{D}$ can be seen as consisting of several full copies of $\mathcal{C}$ and $\# \mathcal{C}$ divides $\# \mathcal{D}$. They have the same size if and only if, once fixed some state $\mathbf{u}$ of $\mathcal{C}$, for any different states $x, y \in Q$, $\mathbf{u} x$ and $\mathbf{u} y$ cannot both belong to $\mathcal{D}$.

If from a connected component $\mathcal{C}$ of $\mathcal{A}^{n}$, we obtain several connected components of $\mathcal{A}^{n+1}$, we say that $\mathcal{C}$ splits up.

The connected components of the powers of a Mealy automaton and the questions of the finiteness of the generated group or the existence of a monogenic subgroup are closely related, as shown in the following propositions (obtained also independently in [7]).

- Proposition 6. An invertible-reversible Mealy automaton generates a finite group if and only if the connected components of its powers have bounded size.

Proof. Let $\mathcal{A}$ be an invertible-reversible Mealy automaton.

Let $\mathcal{C}$ be a connected component of some $\mathcal{A}^{n}$, and $\mathbf{u}, \mathbf{v}$ two states of $\mathcal{C}$ : because of the reversibility of $\mathcal{A}^{n}, \mathbf{v}$ is the image of $\mathbf{u}$ by the action of an element of $\langle\mathfrak{d}(\mathcal{A})\rangle$. If $\mathcal{A}$ generates a finite group, so does $\mathfrak{d}(\mathcal{A})$ by Proposition 3 , and hence $\# \mathcal{C}$ is bounded by $\#\langle\mathfrak{d}(\mathcal{A})\rangle$.

Conversely, if the connected components of the powers of $\mathcal{A}$ have bounded size, as there is only a finite number of Mealy automata of bounded size, there exist $p<q$ such that $\mathfrak{m}\left(\mathcal{A}^{p}\right)$ and $\mathfrak{m}\left(\mathcal{A}^{q}\right)$ are isomorphic, and therefore $\mathcal{A}$ generates a finite group from Lemma 4 .

- Proposition 7. Let $\mathcal{A}=(Q, \Sigma, \delta, \rho)$ be an invertible-reversible Mealy automaton and let $\mathbf{u} \in Q^{+}$be a non-empty word. The following conditions are equivalent:

(i) $\rho_{\mathbf{u}}$ has finite order,

(ii) the sizes of the connected components of $\left(\mathbf{u}^{n}\right)_{n \in \mathbb{N}}$ are bounded,

(iii) there exists a word $\mathbf{v}$ such that the sizes of the connected components of $\left(\mathbf{v} \mathbf{u}^{n}\right)_{n \in \mathbb{N}}$ are bounded,

(iv) for any word $\mathbf{v}$, the sizes of the connected components of $\left(\mathbf{v u}^{n}\right)_{n \in \mathbb{N}}$ are bounded.

Proof. (iii) $\Rightarrow$ (iii), (iv) $\Rightarrow$ (ii), and (iv) $\Rightarrow$ (iii) are immediate.

(ii) $\Rightarrow$ (ii) is a direct consequence of Proposition 6. let $k$ be the order of $\rho_{\mathbf{u}}$; it means that $\mathbf{u}^{k}$ acts as the identity, and so do all the states of its connected component. By Proposition 6 , the connected components of the $\left(\mathbf{u}^{k n}\right)_{n}$ have bounded size, which leads to (iii).

(iii) $\Rightarrow$ (ii): for each $n$, denote by $\mathcal{C}_{n}$ the connected component of $\mathbf{v u}^{n}$. As the sizes of these components are bounded, the sequence $\left(\mathcal{C}_{n}\right)_{n}$ admits a subsequence which all elements are the same, up to state numbering. Within this subsequence, there are two elements such that two different words $\mathbf{v u}^{*}$ name the same state, say $\mathbf{v} \mathbf{u}^{p}$ and $\mathbf{v} \mathbf{u}^{q}$, which means that $\rho_{\mathbf{v} \mathbf{u}^{p}}=\rho_{\mathbf{v} \mathbf{u}^{q}}$, and $\rho_{\mathbf{u}}$ has finite order.

(iii) $\Rightarrow$ (iv): the size of the connected component of $\mathbf{v} \mathbf{u}^{n}$ is at most $\# \Sigma^{|\mathbf{v}|}$ times the size of the connected component of $\mathbf{u}^{n}$. 


\section{The Labeled Orbit Tree}

In this section, we build a tree capturing the links between the connected components of consecutive powers of a Mealy automaton. An example of the first levels of such a tree is given in Figure 3 ,

Let $\mathcal{A}=(Q, \Sigma, \delta, \rho)$ be an invertible-reversible Mealy automaton. Consider the tree with vertices the connected components of the powers of $\mathcal{A}$, and the incidence relation built by adding an element of $Q$ : for any $n \geq 0$, the connected component of $\mathbf{u} \in Q^{n}$ is linked to the connected component(s) of $\mathbf{u} x$, for any $x \in Q$. This tree is called the orbit tree of $\mathfrak{d}(\mathcal{A})[9]$. It can be seen as the quotient of the tree $Q^{*}$ under the action of the group $\langle\mathfrak{d}(\mathcal{A})\rangle$.

We label any edge $\mathcal{C} \rightarrow \mathcal{D}$ of the orbit tree by the ratio $\frac{\# \mathcal{D}}{\# \mathcal{C}}$, which is always an integer by the reversibility of $\mathcal{A}$. We call this labeled tree the labeled orbit tree of $\mathfrak{d}(\mathcal{A})$. In $[9]$, in the definition of the labeled orbit tree, each vertex is labeled by the size of the associated connected component, which encodes exactly the same information as our relative labeling, as the root has size one. We denote by $\mathfrak{t}(\mathcal{A})$ the labeled orbit tree of $\mathfrak{d}(\mathcal{A})$. Note that for each vertex of $\mathfrak{t}(\mathcal{A})$ the sum of the labels of all edges going down from this vertex always equals to the number of states in $\mathcal{A}$.

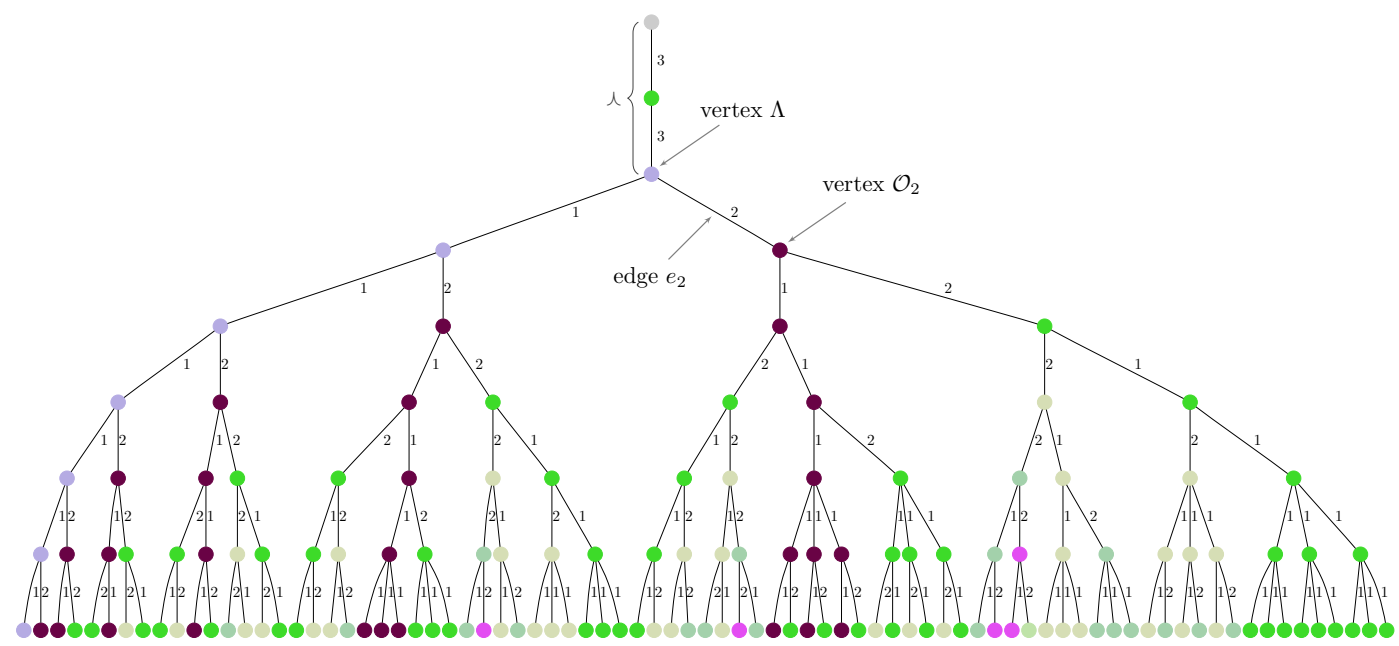

Figure 3 The labeled orbit tree of the Mealy automaton of Figure 1 (up to level 7).

From now on we will assume that all the orbit trees are labeled.

Let $\mathbf{u}$ be a (possibly infinite) word over $Q$. The path of $\mathbf{u}$ in the orbit tree $\mathfrak{t}(\mathcal{A})$ is the unique initial path going from the root through the connected components of the prefixes of $\mathbf{u} ; \mathbf{u}$ can be called a representative of this initial path (or of the orbit of $\mathbf{u}$ under $\langle\mathfrak{d}(\mathcal{A})\rangle$ representing the endpoint of this path); we can say equivalently that this path is represented by $\mathbf{u}$.

- Definition 8. Let $e$ and $f$ be two edges in the orbit tree $\mathfrak{t}(\mathcal{A})$. We say that $e$ is liftable to $f$ if each word of $\perp(e)$ admits some word of $\perp(f)$ as a suffix.

Obviously if $e$ is liftable to $f$, then $f$ is closer to the root of the orbit tree. The fact that an edge is liftable to another one reflects a deeper relation stated below. The following lemma is one of the key observations that we use many times later in the paper.

- Lemma 9. Let $e$ and $f$ be two edges in the orbit tree $\mathfrak{t}(\mathcal{A})$. If $e$ is liftable to $f$, then the label of $e$ is less than or equal to the label of $f$. 
Proof. Since $e$ is liftable to $f$, each word in $\perp(e)$ has a form $\mathbf{v u} x$ for some $\mathbf{u} x \in \perp(f)$. Suppose that $\mathbf{v u} x$ and $\mathbf{v u} y$ are in the same connected component: there exists $\mathbf{s} \in \Sigma^{*}$ such that $\delta_{\mathbf{s}}$ moves $\mathbf{v u} x$ to $\mathbf{v u} y$. In this case, $\rho_{\mathbf{v}}(\mathbf{s})$ moves $\mathbf{u} x$ to $\mathbf{u} y$. Thus, the number of children of $\mathbf{v u}$ in the connected component of $\mathbf{v u} x$ (which is equal to the label of $e$ ) is less than or equal to the number of children of $\mathbf{u}$ in the connected component of $\mathbf{u} x$ (which is equal to the label of $f$ ).

This notion can be generalized to paths:

- Definition 10. Let $\mathbf{e}=\left(e_{i}\right)_{i \in I}$ and $\mathbf{f}=\left(f_{i}\right)_{i \in I}$ be two paths of the same (possibly infinite) length in the orbit tree $\mathfrak{t}(\mathcal{A})$. The path $\mathbf{e}$ is liftable to the path $\mathbf{f}$ if, for any $i \in I$, the edge $e_{i}$ is liftable to the edge $f_{i}$.

As each word $\mathbf{u} \in Q^{*}$ is a state in a connected component of $\mathcal{A}^{|\mathbf{u}|}$, we can notice the following fact which is crucial for all our forthcoming proofs.

- Lemma 11. Let $\mathbf{e}$ be a path at level $k$ in the orbit tree $\mathfrak{t}(\mathcal{A})$. Then, for any $\ell<k$, e is liftable to some path at level $\ell$. In particular, e is liftable to some initial path.

\section{$5 \quad$ Main result}

We study here the case where $\mathcal{A}$ is a connected invertible-reversible 3 -state Mealy automaton, which means that the orbit tree of its dual has a unique edge adjacent to the root, labeled by 3. We prove that if $\mathcal{A}$ generates an infinite group, then the orbit tree $\mathfrak{t}(\mathcal{A})$ admits a (necessarily unique) branch without edges labeled by 1 , more precisely a branch of label either $3^{\omega}$ or $3^{n} 2^{\omega}$, where $i^{\omega}$ denotes an infinite word whose each letter is $i$. An element of infinite order will then be constructed using this branch.

The restriction to connected Mealy automata is discussed in Remark 28

\subsection{General structure of the orbit tree}

From Lemma 11, we obtain the following result on the connection degree of a Mealy automaton (note that it does not depend on the number of states in the Mealy automaton):

- Proposition 12. If for some n, a connected component of $\mathcal{A}^{n}$ does not split up, then the connection degree of $\mathcal{A}$ is at least $n+1$.

Proof. Suppose that $\mathcal{A}$ has $m$ states. If an edge at level $n$ in the orbit tree $\mathfrak{t}(\mathcal{A})$ has label $m$, by Lemma 11, it is liftable to some edge at any level above $n$, and by Lemma 9 this edge is labeled by $m$. Now, by going from top to bottom, we can conclude that there is only one edge at each level above $n+1$ in the orbit tree.

Now we restrict our attention again on the case of 3-state automata, unless specified otherwise. If the connection degree of such an automaton $\mathcal{A}$ is infinite, it has been proved in [17, Proposition 14] that the generated semigroup is free, freely generated by the states of the Mealy automaton. As a consequence $\mathcal{A}$ cannot generate an infinite Burnside group in this case. So from now on, we assume $0<\curlywedge(\mathcal{A})<\infty$.

We know now that the orbit tree $\mathfrak{t}(\mathcal{A})$ has a prefix linear part until level $\curlywedge(\mathcal{A})$ and that below this level, all the vertices split up. We denote by $\Lambda(\mathcal{A})$ the highest vertex to split up (i.e. the only vertex at level $\curlywedge(\mathcal{A})$ ). 
- Definition 13. Let $\mathbf{i}$ be a (possibly infinite) word over an alphabet $F$ and let $j \in F$. A $j$-block $\mathbf{j}$ of $\mathbf{i}$ is a maximal factor of $\mathbf{i}$ in $j^{*} \cup\left\{j^{\omega}\right\}$, that is, $\mathbf{i}=\mathbf{k j l}$ holds, where the last letter of $\mathbf{k}$ and the first letter of $\mathbf{l}$, if not empty, are not $j$.

- Lemma 14. If the lengths of the 2-blocks in the orbit tree $\mathfrak{t}(\mathcal{A})$ are not bounded, then $\mathfrak{t}(\mathcal{A})$ admits a branch labeled by $3^{\curlywedge(\mathcal{A})} 2^{\omega}$. If the lengths of the 2-blocks are bounded with supremum $N$, then $\mathfrak{t}(\mathcal{A})$ admits an initial path labeled by $3^{\curlywedge(\mathcal{A})} 2^{N}$ (and none labeled by $3^{\curlywedge(\mathcal{A})} 2^{N+1}$ ).

Proof. As there is at most one path starting at $\Lambda(\mathcal{A})$ with a maximal prefix in $2^{\omega}$ because the stateset has size 3, Lemma 11 leads to the conclusion.

By Proposition 12, below the connection degree of $\mathcal{A}$, no edge can be labeled by 3 . On the other hand, the case when all edges are labeled by 1 turns out to be irrelevant for generating infinite Burnside groups,according to the following proposition (that holds for automata with arbitrary number of states).

- Proposition 15. If all edges coming down from the only connected component at vertex $\Lambda(\mathcal{A})$ are labeled by 1 , the group generated by $\mathcal{A}$ is finite.

Proof. By Lemmas 9 and 11, below level $\curlywedge(\mathcal{A})$, all edges are then labeled by 1 , which means that the connected components of the powers of $\mathcal{A}$ have bounded size, and the group $\langle\mathcal{A}\rangle$ is finite according to Proposition 6 .

From now on, we assume that the only connected component at vertex $\Lambda(\mathcal{A})$ splits up in two connected components.

\subsection{Reduction edge and orbital words}

Let us recall our framework: $\mathcal{A}$ is a connected 3 -state invertible-reversible Mealy automaton with $0<\curlywedge(\mathcal{A})<\infty$, such that the only connected component at vertex $\Lambda(\mathcal{A})$ splits up in two connected components. The point is now to put the emphasize on the larger of the two.

- Definition 16. At level $\curlywedge(\mathcal{A})$ there are two edges, one labeled by 1 and a second one labeled by 2 . We call by the reduction edge this last edge and denote it by $e_{2}$, and denote by $\mathcal{O}_{2}$ its terminal vertex, and call it the reduction orbit.

- Lemma 17. Each vertex below the level $\curlywedge(\mathcal{A})+1$ is the initial vertex of either one edge which is liftable to the reduction edge and one edge which is not, or two edges which are liftable to the reduction edge and one which is not.

- Definition 18. An $e_{2}$-liftable path is an initial (possibly infinite) path in the orbit tree whose edges below the level $\curlywedge(\mathcal{A})$ are all liftable to the reduction edge $e_{2}$ of $\mathfrak{t}(\mathcal{A})$.

- Definition 19. A word over $Q$ is said to be orbital if it is a representative of an $e_{2}$-liftable path or, equivalently, if all its length $\curlywedge(\mathcal{A})$ factors belong to the reduction orbit $\mathcal{O}_{2}$. Denote then by $T_{\mathcal{O}_{2}}$ the set of all finite orbital words. Being prefix-closed, $T_{\mathcal{O}_{2}}$ can be seen either as a set of words, or as a tree.

Note that $T_{\mathcal{O}_{2}}$ is a strictly $(\curlywedge(\mathcal{A})+1)$-testable language [18. In particular, for any word $\mathbf{u} \in$ $T_{\mathcal{O}_{2}}$ of length at least $\curlywedge(\mathcal{A})$, the set of words $\mathbf{v}$ such that $\mathbf{u v}$ belongs to $T_{\mathcal{O}_{2}}$ depends only on the length $\curlywedge(\mathcal{A})$ suffix of $\mathbf{u}$. A simple consequence is that $T_{\mathcal{O}_{2}}$ viewed as a set of infinite words is factor-closed. Further, up to level $\curlywedge(\mathcal{A})$, the tree $T_{\mathcal{O}_{2}}$ coincides with $Q^{*}$ and each word of length at least $\curlywedge(\mathcal{A})$ is a prefix of exactly two other words in $T_{\mathcal{O}_{2}}$. 
We now state several technical results about the tree $T_{\mathcal{O}_{2}}$ of orbital words which will be of use in the final subsection.

- Lemma 20. For any two words $\mathbf{u}, \mathbf{v}$ in $T_{\mathcal{O}_{2}}$ and any integer $n$, there exists a word $\mathbf{r} \in Q^{n}$ satisfying ur $\in T_{\mathrm{O}_{2}}$ and $\mathbf{v r} \in T_{\mathrm{O}_{2}}$.

Proof. For any word $\mathbf{w} \in T_{\mathcal{O}_{2}}$, there exist (at least) two different states $q_{1}, q_{2} \in Q$ satisfying $\mathbf{w} q_{1}, \mathbf{w} q_{2} \in T_{\mathcal{O}_{2}}$. As the stateset $Q$ has size 3 , there is at least one common state $r_{1} \in Q$ satisfying $\mathbf{u} r_{1} \in T_{\mathcal{O}_{2}}$ and $\mathbf{v} r_{1} \in T_{\mathcal{O}_{2}}$. The result is then obtained by recursion.

- Lemma 21. Let $x \in Q$ and $\mathbf{u} \in Q^{\curlywedge(\mathcal{A})}$. The set of length $\curlywedge(\mathcal{A})$ suffixes of all words in the connected component of $x \mathbf{u}$ is the whole $Q^{\curlywedge(\mathcal{A})}$.

Proof. Since $\mathcal{A}$ is reversible and $\mathcal{A}^{\curlywedge(\mathcal{A})}$ connected, for each $\mathbf{v} \in Q^{\curlywedge(\mathcal{A})}$, there exists $\mathbf{s} \in \Sigma^{*}$ such that $\delta_{\mathbf{s}}(\mathbf{u})=\mathbf{v}$. By the invertibility of $\mathcal{A}, \mathbf{t}=\rho_{x}^{-1}(\mathbf{s})$ is well defined and we have

$$
\delta_{\mathbf{t}}(x \mathbf{u})=\delta_{\mathbf{t}}(x) \delta_{\rho_{x}(\mathbf{t})}(\mathbf{u})=\delta_{\mathbf{t}}(x) \delta_{\mathbf{s}}(\mathbf{u})=\delta_{\mathbf{t}}(x) \mathbf{v} .
$$

Proposition 22. For any orbital word $\mathbf{u}$, there are infinitely many edges in $T_{\mathcal{O}_{2} \mid \mathbf{u}}$ labeled by each state.

Proof. By contradiction. Denote the stateset $Q=\{x, y, z\}$ and let $\mathbf{u}$ be an orbital word (of length at least $\curlywedge(\mathcal{A})$ ) such that no edge of $T_{\mathcal{O}_{2} \mid \mathbf{u}}$ is labeled by $z$.

As each word of $T_{\mathcal{O}_{2}}$ can be extended in $T_{\mathcal{O}_{2}}$ by two different states, $x$ and $y$ are in $T_{\mathcal{O}_{2} \mid \mathbf{u}}$. By recursion, $\{x, y\}^{*}$ is a subset of $T_{\mathcal{O}_{2} \mid \mathbf{u}}$ and, as $T_{\mathcal{O}_{2}}$ is suffix-closed, $\{x, y\}^{*} \subseteq T_{\mathcal{O}_{2}}$. Let $\mathbf{v} \in$ $\{x, y\}^{\curlywedge(\mathcal{A})-1}: x \mathbf{v}$ and $y \mathbf{v}$ are elements of $T_{\mathcal{O}_{2}}$ and $x \mathbf{v} x, x \mathbf{v} y, y \mathbf{v} x$, and $y \mathbf{v} y$ are in $\mathcal{O}_{2}$. Hence $x \mathbf{v} z$ and $y \mathbf{v} z$ have length $\curlywedge(\mathcal{A})+1$ and are not in $\mathcal{O}_{2}$. In this case both these words must belong to the other connected component of size $3^{\curlywedge(\mathcal{A})}$.

By Lemma 21, the connected component of $x \mathbf{v} z$ will have at least $3^{\curlywedge}(\mathcal{A})$ words with different suffixes starting from position 2. Therefore, if we assume that $y \mathbf{v} z$ is also in this component, we must have at least $3^{\curlywedge(\mathcal{A})}+1$ words in there. Contradiction.

\subsection{Cyclically orbital words and elements of infinite order}

In this subsection, in the case when the lengths of the 2-blocks in the orbit tree are bounded, we exhibit a family of words whose induced actions have finite bounded orders. Then we prove that each word admits a bounded power which induces the same action as some word in this family.

- Definition 23. A word over $Q$ is said to be cyclically orbital if each of its powers is orbital. In other words, a word is cyclically orbital if viewed as a cyclic word it is orbital.

Note that the existence of such cyclically orbital words is ensured by the straightforward fact that any orbital word of length $\curlywedge(\mathcal{A}) \times\left(1+\# Q^{\curlywedge(\mathcal{A})}\right)$ admits a cyclically orbital factor.

To prove the first result about cyclically orbital words we will need the following proposition.

- Proposition 24. If the lengths of the 2-blocks in $\mathfrak{t}(\mathcal{A})$ are bounded with supremum $N$, then each edge from level $\curlywedge(\mathcal{A})+N$ or below in an $e_{2}$-liftable path is followed by three edges. 
Proof. From Lemma 14 there is an initial path labeled by $3^{\curlywedge(\mathcal{A})} 2^{N}$ (and none labeled by $\left.3^{\curlywedge(\mathcal{A})} 2^{N+1}\right)$. As the stateset has size 3 , this path is unique, call it $\mathbf{e}: \perp(\mathbf{e})$ is the initial vertex of 3 edges, two of which are liftable to the reduction edge by Lemma 17

We now consider the subtree $\mathfrak{s}$ of $\mathfrak{t}(\mathcal{A})$ consisting of all $e_{2}$-liftable paths. By Lemma 17, each path of $\mathfrak{s}$ is either a prefix of $\mathbf{e}$ or prefixed with $\mathbf{e}$. Suppose that one of the edges of $\mathfrak{s}$ below level $|\mathbf{e}|+1$ is labeled by 2 and consider an initial branch $\mathbf{f}$ in $\mathfrak{s}$ which minimizes the length of the 1-block from $\perp(\mathbf{e})$ : its label has prefix $3^{\curlywedge(\mathcal{A})} 2^{N} 1^{k} 2$ for some $k>0$. Consider the (noninitial) path in $\mathfrak{t}(\mathcal{A})$ obtained from $\mathbf{f}$ by erasing the first edge: by Lemma 11 it is liftable to an initial path, say $\mathbf{g}$. As $\mathbf{f}$ is an $e_{2}$-liftable path, so is $\mathbf{g}$, hence $\mathbf{g}$ coincides necessarily with $\mathbf{e}$ until level $\curlywedge(\mathcal{A})+N$; so the label of $\mathbf{g}$ has prefix $3^{\curlywedge(\mathcal{A})} 2^{N}$. By Lemmas 9 and 11, it has also a prefix whose label is greater than or equal to (coordinatewise) $3^{\curlywedge(\mathcal{A})-1} 2^{N} 1^{k} 2$. Hence the label of $\mathbf{g}$ has a prefix greater than or equal to $3^{\curlywedge(\mathcal{A})} 2^{N} 1^{k-1} 2$, which is in contradiction with the choice of $\mathbf{f}$.

- Proposition 25. If the lengths of the 2-blocks in $\mathfrak{t}(\mathcal{A})$ are bounded, any cyclically orbital word induces an action of finite order, bounded by a constant not depending on the word.

Proof. Let $\mathbf{u}$ be a cyclically orbital word and $n$ be an integer such that $\left|\mathbf{u}^{n}\right|>\curlywedge(\mathcal{A})$. By the definition, $\mathbf{u}^{n}$ is a representative of some $e_{2}$-liftable path. So, by Lemma 14, the label of the path of $\mathbf{u}^{\omega}$ is ultimately 1 and, by Proposition 6 , the action induced by $\mathbf{u}$ has finite order, bounded by a constant which depends on $\curlywedge(\mathcal{A})$ (more precisely on how many ways one can choose the outputs for a Mealy automaton with the same structure as $\perp$ (e) where $\mathbf{e}$ is the path of $\mathfrak{t}(\mathcal{A})$ defined in the proof of Proposition 24).

- Proposition 26. If the lengths of the 2-blocks in $\mathfrak{t}(\mathcal{A})$ are bounded, any non-empty word over $Q$ admits a non-empty bounded power which is equivalent to a cyclically orbital word.

Proof. Let $\curlywedge=\curlywedge(\mathcal{A})$ be the connection degree of $\mathcal{A}$. For a (possibly infinite) word $\mathbf{w} \in T_{\mathcal{O}_{2}}$, let fact $(\mathbf{w})$ denote the (finite) set of its length $\curlywedge$ factors.

Consider an infinite word $\mathbf{u} \in T_{\mathcal{O}_{2}}$, that we assume to be maximal in the sense that there is no other infinite word $\mathbf{u}^{\prime} \in T_{\mathcal{O}_{2}}$ satisfying fact ${ }_{\curlywedge}(\mathbf{u}) \varsubsetneqq$ fact $_{\curlywedge}\left(\mathbf{u}^{\prime}\right)$. Let fix a finite prefix $\mathbf{v}$ of $\mathbf{u}$ satisfying fact $\mathrm{f}_{\curlywedge}(\mathbf{u})=$ fact $_{\curlywedge}(\mathbf{v})$. From the maximality assumption on $\mathbf{u}$, we deduce that each $\mathbf{w} \in T_{\mathcal{O}_{2} \mid \mathbf{v}}$ (where $T_{\mathcal{O}_{2} \mid \mathbf{v}}$, viewed as a set of words, consists of words $\mathbf{w}$ over $Q$ such that $\left.\mathbf{v w} \in T_{\mathcal{O}_{2}}\right)$ satisfies fact $(\mathbf{w}) \subseteq$ fact $_{\curlywedge}(\mathbf{v})$. We will refer to this property as Property $(\mathfrak{\natural )}$.

Let $a_{1} a_{2} \ldots a_{n} \in Q^{+}$. By Proposition 22 $a_{1}$ appears infinitely many often in the tree $T_{\mathcal{O}_{2} \mid \mathbf{v}}$. Therefore there is some word $\mathbf{u}_{0}$ satisfying $\mathbf{u}_{0} a_{1} \in T_{\mathcal{O}_{2} \mid \mathbf{v}}$. The goal is to build a word $\mathbf{u}_{1}$ satisfying $\mathbf{u}_{\mathbf{0}} a_{1} \mathbf{u}_{\mathbf{1}} a_{2} \in T_{\mathcal{O}_{2} \mid \mathbf{v}}$. As in Figure 4, choose some word $\mathbf{v}_{\mathbf{0}}$ satisfying $\mathbf{v}_{\mathbf{0}} a_{2} \in T_{\mathcal{O}_{2} \mid \mathbf{v}}$. By Lemma 20 and Property ( $($ ) $)$, there exists a word $\mathbf{r} \in$ fact $_{\curlywedge}(\mathbf{v})$ such that both $\mathbf{u}_{\mathbf{0}} a_{1} \mathbf{r} \in$ $T_{\mathcal{O}_{2} \mid \mathbf{v}}$ and $\mathbf{v}_{\mathbf{0}} \mathbf{r} \in T_{\mathcal{O}_{2} \mid \mathbf{v}}$ hold. Let $\mathbf{v}_{\mathbf{r}}$ be the shortest word such that $\mathbf{v}_{\mathbf{r}} \mathbf{r}$ is a prefix of $\mathbf{v}$ and let $\mathbf{u}_{\mathbf{1}}^{\prime}$ be the word satisfying $\mathbf{v}_{\mathbf{r}} \mathbf{u}_{\mathbf{1}}^{\prime}=\mathbf{v v}_{\mathbf{0}}$. Then $\mathbf{u}_{\mathbf{1}}^{\prime}$ is a cyclically orbital word, since its length $\curlywedge$ prefix $\mathbf{r}$ satisfies $\mathbf{v}_{\mathbf{r}} \mathbf{u}_{1}^{\prime} \mathbf{r} \in T_{\mathcal{O}_{2}}$.

By Proposition 25, $\mathbf{u}_{\mathbf{1}}^{\prime}$ has finite order $q$. So we set $\mathbf{u}_{\mathbf{1}}=\mathbf{u}_{\mathbf{1}}^{\prime}{ }^{q}$ and keep $a_{1} \mathbf{u}_{1} a_{2} \in T_{\mathcal{O}_{2} \mid \mathbf{v} \mathbf{u}_{0}}$. The same method produces words $\left(\mathbf{u}_{\mathbf{i}}\right)_{1 \leq \mathbf{i} \leq n}$ of length at least $\curlywedge$ that induce the trivial action and such that the word $\mathbf{w}^{(\mathbf{0})}=a_{1} \mathbf{u}_{\mathbf{1}} a_{2} \cdots a_{n-1} \mathbf{u}_{\mathbf{n}-\mathbf{1}} a_{n} \mathbf{u}_{\mathbf{n}}$ satisfies $\mathbf{w}^{(\mathbf{0})} a_{1} \in T_{\mathcal{O}_{2} \mid \mathbf{v u}_{\mathbf{0}}}$ and induces the same action as $a_{1} \cdots a_{n}$. For $i \geq 0$, we analogously define a word $\mathbf{w}^{(\mathbf{i}+\mathbf{1})}$ inducing the same action as $a_{1} \cdots a_{n}$ such that $\mathbf{w}^{(\mathbf{i}+\mathbf{1})} a_{1} \in T_{\mathcal{O}_{2} \mid \mathbf{v u}_{\mathbf{0}} \mathbf{w}^{(\mathbf{0}) \cdots} \mathbf{w}^{(\mathbf{i})}}$. Eventually, there exist $\mathbf{i}<\mathbf{j}$ such that $\mathbf{u}_{\mathbf{1}}^{(\mathbf{i})}$ and $\mathbf{u}_{\mathbf{1}}^{(\mathbf{j}+\mathbf{1})}$ have the same prefix of length $\curlywedge$, hence $\mathbf{w}^{(\mathbf{i})} \cdots \mathbf{w}^{(\mathbf{j})}$ is cyclically orbital and induces the same action as $\left(a_{1} \cdots a_{n}\right)^{\mathbf{j}-\mathbf{i}}$. Note that $\mathbf{j}-\mathbf{i}$ is bounded by a constant depending on $\curlywedge(\mathcal{A})$ and $\# Q$. 


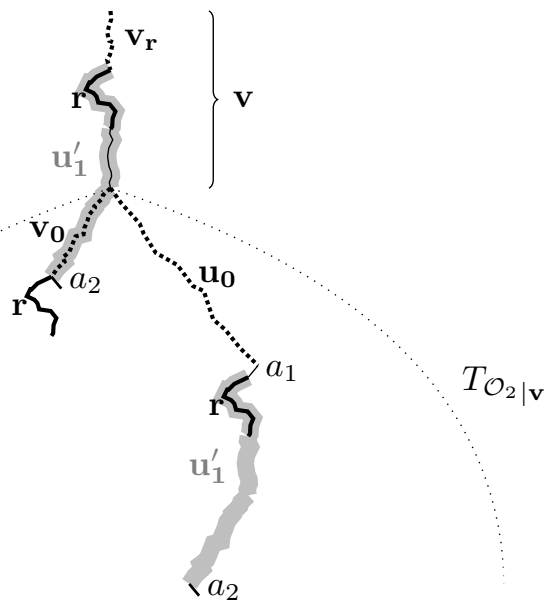

Figure 4 Proof of Proposition 26, building a word $\mathbf{u}_{1}^{\prime}$ satisfying $\mathbf{u}_{\mathbf{0}} a_{1} \mathbf{u}_{\mathbf{1}}^{\prime} a_{2} \in T_{\mathcal{O}_{2} \mid \mathbf{v}}$.

- Corollary 27. If $\mathcal{A}$ generates an infinite group, the orbit tree of its dual admits an $e_{2}$ liftable branch labeled either by $3^{\omega}$ or by $3^{n} 2^{\omega}$ for some $n$.

Proof. If the lengths of the 2-blocks are bounded, any non-empty word has a non-empty bounded power which is equivalent to a cyclically orbital word by Proposition 26, so the order of the action it induces is finite and bounded by a constant from Propositions 25$]$ and 26 .

It follows from Zelmanov's solution to the restricted Burnside problem [23, 24, 25] that the group $\langle\mathcal{A}\rangle$ is finite, which contradicts the hypothesis. Proposition 24leads to the conclusion.

- Remark 28. A disconnected 3-state invertible-reversible Mealy automaton have either three connected components - each of size 1 - and the generated group is finite (see Proposition [15), or two connected components - of size 1 and 2 - and the situation is then more subtle because both connected components can generate separately finite groups, but together an infinite group. Such a disconnected Mealy automaton might not satisfy Corollary 27. For example, the Mealy automaton in Figure2 2 illustrates this situation. The group that it generates is infinite since the product of two components of this automaton generates an infinite subgroup $\langle x y, x z\rangle$ [1, 17]. However, if there is an initial branch labeled by $2^{\omega}$ and the analogue of Corollary 27 holds, it would imply that both $y$ and $z$ have infinite order, which is not the case as $\langle y, z\rangle$ is finite.

- Theorem 29. Any infinite group generated by a connected 3-state invertible-reversible Mealy automaton admits an element of infinite order.

Proof. All cyclically orbital words induce actions of infinite order from Proposition 7 and Corollary 27

Finally we can emphasize that the proof of existence of elements of infinite order is constructive and that the effective detection of such elements becomes computable in this framework. For instance, in the group generated by the Mealy automaton in Figure 1, the cyclically orbital word $x y z$ has infinite order. At least in this case, neither one of the existing two packages FR [3] and automgrp [19] for GAP [8] system, dedicated to Mealy automata and groups they generate, is able to detect such an element of infinite order. 


\section{References}

1 A. Akhavi, I. Klimann, S. Lombardy, J. Mairesse, and M. Picantin, On the finiteness problem for automaton (semi)groups, Int. J. Algebra Comput. 22 (2012), no. 6, 26p.

2 S.V. Alešin, Finite automata and the Burnside problem for periodic groups, Mat. Zametki 11 (1972), 319-328.

3 L. Bartholdi, FR functionally recursive groups, self-similar groups - a gap package, version 2.1.1, 2014

4 L. Bartholdi and Z. Šunik, On the word and period growth of some groups of tree automorphisms, Comm. Algebra 29 (2001), no. 11, 4923-4964.

5 James Belk and Collin Bleak, Some undecidability results for asynchronous transducers and the Brin-Thompson group 2V, Preprint: arxiv:1405.0982, 2014.

6 W. Burnside, On an unsettled question in the theory of discontinuous groups, Quart.J.Math. 33 (1902), 230-238.

7 D. D'Angeli and E. Rodaro, A geometric approach to (semi)-groups defined by automata via dual transducers, Preprint: arxiv:1403.1722, 2014.

8 The GAP Group, GAP - Groups, Algorithms, and Programming, Version 4.7.5, 2014.

9 P.W. Gawron, V.V. Nekrashevych, and V.I. Sushchansky, Conjugation in tree automorphism groups, Internat. J. Algebra Comput. 11 (2001), no. 5, 529-547.

10 P. Gillibert, The finiteness problem for automaton semigroups is undecidable, Internat. J. Algebra Comput. 24 (2014), no. 1, 1-9.

11 V.M. Gluškov, Abstract theory of automata, Uspehi Mat. Nauk 16 (1961), no. 5 (101), $3-62$.

12 E.S. Golod, On nil-algebras and finitely residual groups, Izv. Akad. Nauk SSSR. Ser. Mat. 28 (1964), 273-276.

13 E.S. Golod and I. Shafarevich, On the class field tower, Izv. Akad. Nauk SSSR Ser. Mat. 28 (1964), 261-272.

14 R.I. Grigorchuk, On Burnside's problem on periodic groups, Funktsional. Anal. i Prilozhen. 14 (1980), no. 1, 53-54.

15 , Degrees of growth of finitely generated groups and the theory of invariant means, Izv. Akad. Nauk SSSR Ser. Mat. 48 (1984), no. 5, 939-985.

16 N. Gupta and S. Sidki, On the Burnside problem for periodic groups, Math. Z. 182 (1983), no. $3,385-388$.

17 I. Klimann, The finiteness of a group generated by a 2-letter invertible-reversible Mealy automaton is decidable, Proc. 30th STACS, LIPIcs, vol. 20, 2013, pp. 502-513.

18 R. Mac Naughton and S. Papert, Counter free automata, The MIT Press, 1971.

19 Y. Muntyan and D. Savchuk, automgrp automata groups - a gap package, version 1.2.4, 2014 .

20 V. Nekrashevych, Self-similar groups, Mathematical Surveys and Monographs, vol. 117, American Mathematical Society, Providence, RI, 2005.

21 D.M. Savchuk and Y. Vorobets, Automata generating free products of groups of order 2 , J. Algebra 336 (2011), no. 1, 53-66.

22 V.I. Sushchansky, Periodic permutation p-groups and the unrestricted Burnside problem, DAN SSSR. 247 (1979), no. 3, 557-562, (in Russian).

23 M. Vaughan-Lee, The restricted Burnside problem, London Mathematical Society Monographs. New Series, vol. 8, Oxford University Press, 1993.

24 E.I. Zel'manov, Solution of the restricted Burnside problem for groups of odd exponent, Izv. AN SSSR Math+ 54 (1990), no. 1, 42-59, 221.

25 , Solution of the restricted Burnside problem for 2-groups, Mat. Sb. 182 (1991), no. $4,568-592$. 